\title{
Verwandtschaften, Wahlverwandtschaften und andere Beziehungskisten: Deutschdidaktik als transdisziplinäres Fach
}

\author{
Anita Schilcher $\cdot$ Christel Meier
}

Angenommen: 7. Januar 2021 / Online publiziert: 17. Februar 2021

(C) Der/die Autor(en) 2021

Zusammenfassung Deutschdidaktische Forschung hat sich seit dem PISA-Schock Anfang des Jahrtausends wesentlich verändert. Während traditionell ein starker Bezug zu den germanistischen Fachwissenschaften besteht, kann deutschdidaktische Forschung der letzten Jahre als domänenspezifische Bildungswissenschaft mit zahlreichen Bezügen zu unterschiedlichen Disziplinen charakterisiert werden, wobei die Rückbindung an die Germanistik gerade für den bildungswissenschaftlichen Diskurs von entscheidender Bedeutung ist.

Schlüsselwörter Transdisziplinarität · Interdisziplinarität · Fachdidaktik · Deutschdidaktik · Kompetenzorientierung · Erklären als Lehrkraftkompetenz • Literarische Textverstehenskompetenz

\footnotetext{
A. Schilcher $(\square)$

Lehrstuhl für Didaktik der deutschen Sprache und Literatur, Universität Regensburg, Regensburg, Deutschland

E-Mail: anita.schilcher@ur.de

C. Meier

Lehrstuhl für Didaktik der deutschen Sprache und Literatur, Friedrich-Alexander-Universität

Erlangen-Nürnberg, Bismarckstr. 1, 91054 Erlangen, Deutschland

E-Mail: christel.meier@fau.de
} 


\title{
German Education: About Kinships, Elective Affinities and Other Relationships
}

\begin{abstract}
Research in German L1 education has changed significantly since the PISA shock at the beginning of the millennium. While there is traditionally a strong connection between language and literature education and German language and literature studies, German L1 education research in recent years can be characterized as an educational science with numerous references to different disciplines whereby especially for the educational science discourse the connection to German studies is of decisive importance.
\end{abstract}

Keywords Transdisciplinarity - Interdisciplinarity - Subject Matter Education · German L1 Education · Competence Orientation · Explaining as Teacher Competence $\cdot$ Literary Literacy

\section{Deutschdidaktik als transdisziplinäre Wissenschaft}

Wenn die Ergebnisse aus den unterschiedlichen Bildungsstudien (PISA, IGLU, DESI, IQB-Bildungstrend etc.) bedenkliche Ergebnisse zur Leistungsfähigkeit des deutschen Bildungssystems liefern, dann folgt nicht selten eine implizite oder explizite »Schuldzuweisung « an drei Adressatenkreise: Die Lehrkräfte, die Schuladministration oder die Fachdidaktiken. Diese Tatsache mag auf den ersten Blick nicht überraschen, da sich alle drei Akteure mit »Unterricht« auf theoretischer und praktischer Ebene auseinandersetzen. Ob allerdings ein Fach, dessen Anteil am Gesamtstudium (je nach Studiengang und Bundesland) zwischen acht und zwanzig Prozent beträgt, als der entscheidende Einflussfaktor gesehen werden kann, darf bezweifelt werden. Dadurch dass sich in der Forschung zum fachlichen Lernen die fachdidaktische Kompetenz der Lehrkräfte als wichtige Stellschraube erwiesen hat, hat das Nachdenken über die zentralen Inhalte und Aufgaben der Fachdidaktik neuen Auftrieb erhalten.

Die entscheidende Frage, die dabei beantwortet werden muss, ist, welcher Art von Kompetenz es bedarf, um offensichtliche Schwachpunkte des Bildungssystems und der Lehrer/-innenbildung zu beheben. Noch 1995 skizzierte die Hochschulrektorenkonferenz aufgrund mangelnder Studierfähigkeit der Abiturient/-innen eine Leitlinie zur Reform der Lehrer/-innenbildung. Einen Lösungsansatz sah die HRK dabei in der stärkeren Rückbindung der Fachdidaktik an die Schulpraxis. Diese sollte dadurch gewährleistet werden, »daß die Fachdidaktiken nicht durch Professuren auf Lebenszeit vertreten werden, sondern wissenschaftlich qualifizierte, d.h. promovierte, Schulpraktiker nach Ausschreibung und Auswahl durch die Hochschulen für Lehre und Forschung in der Fachdidaktik zeitlich befristet in die Hochschule wechseln« (vgl. KVFF 1998, S. 13).

Probleme in der Praxis sollten durch ein Mehr dieser Praxis an den Universitäten gelöst werden - gestern wie heute eine problematische Schlussfolgerung. Nachdem der erste PISA-Schock überwunden war, wurde wiederum eine andere Konsequenz gezogen: Von der Input- zur Outputorientierung, vom Glauben an die 
Bildungswirksamkeit der Inhalte zur regelmäßigen Überprüfung der Kompetenzen im nationalen und internationalen Vergleich. Auf nationaler Ebene manifestierte sich dies in der Etablierung von Bildungsstandards und in der Ausschreibung zahlreicher Forschungs- und Innovationsprogramme. Wenngleich die Praxisorientierung dabei immer noch eine Rolle spielt, steht die wissenschaftliche Evidenz von unterrichtlichem Handeln für den Lernzuwachs nun stärker im Fokus. Zwei Projekte, die im Rahmen solcher Programme gefördert wurden und den Paradigmenwechsel fachdidaktischer Forschung markieren, werden in diesem Artikel exemplarisch dargestellt.

Dabei stehen zwei Fragen im Mittelpunkt: Wie kann fachdidaktische Forschung zur Steigerung fachbezogener Kompetenzen von Schüler/-innen beitragen und wie muss das Lehramtsstudium gestaltet sein, um einen wesentlichen Beitrag zur Professionalisierung künftiger Lehrkräfte zu leisten? Für beide Aufgaben ist die Fachdidaktik Deutsch in den letzten Jahrzehnten vielfältige Beziehungen zu mehr oder weniger »verwandten « Nachbardisziplinen eingegangen. Diese Kooperationen sollen einleitend im Hinblick auf die Qualitätsoffensive Lehrerbildung und aktuelle Forschungsverbünde skizziert werden, bevor die Überlegungen an den beiden Fallbeispielen zu inter- und transdisziplinären Forschungsprojekten vertieft werden.

\subsection{Die Rolle der Deutschdidaktik für verwandtschaftliche Kooperationen im Kontext der Lehrer/-innenbildung}

Im Rahmen der Qualitätsoffensive Lehrerbildung fördern Bund und Länder in drei unterschiedlichen Förderlinien zwischen 2014 und 2023 universitäre Projekte, die den Prozess der Lehrer/-innenbildung inhaltlich und strukturell nachhaltig verbessern sollen. Ein wesentliches Kriterium der Qualitätsoffensive Lehrerbildung für die Vergabe von Fördermitteln war »eine stärkere Abstimmung all jener an einer Hochschule [...], die für die Ausbildung der Lehrkräfte verantwortlich sind « (BMBF 2019). Die einzelnen Maßnahmen sollten dazu führen, die Inhalte der Ausbildung stärker aufeinander abzustimmen und die Zusammenarbeit zwischen Fachwissenschaft, Fachdidaktik und Erziehungswissenschaft zu verbessern (vgl. ebd.). Wie die mittlerweile erfolgte Umsetzung in der ersten Förderlinie der Qualitätsoffensive in den unterschiedlichen Forschungsprojekten zeigt, sind es meist die Fachdidaktiken, die sich in vielfältigen Projekten entweder mit der Fachwissenschaft (vgl. z. B. SKILL: Strategien zur Kompetenzentwicklung: Innovative Lehr- und Beratungskonzepte in der Lehrerbildung; Universität Passau) oder einer der pädagogischen Bezugswissenschaften (vgl. z. B. EKoL: Effektive Kompetenzdiagnose in der Lehrerbildung; PH Heidelberg und Ludwigsburg) oder auch untereinander (vgl. FALKE: Fachspezifische Lehrerkompetenz im Erklären; Universität Regensburg) vernetzen. Beispiele, in denen sich Fachwissenschaften und Erziehungswissenschaften ohne die Mitwirkung von Fachdidaktiken vernetzt haben, sind kaum zu finden, ebenso wenig wurde unseres Wissens die Qualitätsoffensive Lehrerbildung von germanistischen Fachwissenschaften genutzt, um ein stärker auf die Lehrerbildung zugeschnittenes, kohärentes Studienangebot zu entwickeln, das als das relevante Hintergrundwissen für die Ausbildung einer fachdidaktischen Expertise dienen könnte (vgl. Schilcher/ Krauss 2019). 


\section{Fachwissen Bestimmen und benennen Sie im folgenden Satz die Satzglieder auf Hauptsatzebene. (FW) \\ (Bitte erkennbar segmentieren.)}

Aus pädagogischen Gründen haben die Elternverbände gestern aufs Schärfste kritisiert, dass das Kultusministerium ein Schreiben verfasst hat, in dem Lehrer aufgrund der Schweinegrippe aufgefordert wurden, Schülern künftig nicht mehr die Hand zu geben.

Abb. 1 Aufgabenbeispiel zum Fachwissen aus der FALKO-Studie

Empirische Evidenz für ein Verständnis des Fachwissens als »Hintergrundwissen« lässt sich zum Beispiel aus der COACTIV-Studie, einer an PISA 2003 angedockten Studie zum professionellen Wissen und Handeln von Lehrkräften, ableiten. In COACTIV konnte gezeigt werden, dass das fachdidaktische Wissen der stärkste Prädiktor für die Leistungsentwicklung der Schüler/-innen (im Fach Mathematik) ist. Für das Fachwissen konnten keine vergleichbaren signifikanten Effekte auf Aspekte der Unterrichtsqualität oder auf die Schüler/-innenleistung nachgewiesen werden. Dies heißt jedoch keinesfalls, dass Fachwissen - gefasst als ein tiefes konzeptuelles Verständnis des Unterrichtsstoffes - unwichtig sei. Die Daten legen vielmehr nahe, dass das Fachwissen den Entwicklungsraum des fachdidaktischen Wissens festlegt und damit auch indirekt die Unterrichtsqualität. Auch zahlreiche qualitative Studien haben gezeigt, dass Mängel im Fachwissen die Entwicklungsmöglichkeiten fachdidaktischer Ressourcen limitieren (vgl. hierzu Baumert/Kunter 2011; Krauss et al. 2017, S. 21). »Fachwissen « wird dabei nicht als das akademische, universitäre Fachwissen konzeptualisiert, sondern als vertieftes Hintergrundwissen zum Schulstoff (Krauss et al. 2017, S. 28). Als Beispiel kann ein Item aus der FALKO-Studie (Fachspezifische Lehrerkompetenzen) dienen (siehe Abb. 1).

Dieses literaturwissenschaftliche Item forderte von den Probanden, das Gedicht »Albanie« von Hoffmannswaldau (1616-1679) hinsichtlich seiner prototypischen bzw. abweichenden Merkmale im Vergleich zu barocker Lyrik allgemein zu beschreiben. Die Herausforderung ist dabei, dass die Einordnung des Gedichts zwar das deklarative Wissen über barocke Lyrik in der Tradition der petrarkistischen Liebeslyrik erfordert, jedoch auch darüber hinaus geht, da erkannt werden muss, dass hier die Grenzen dessen, was an erotischen Anspielungen im Barock erlaubt war, deutlich überschritten werden. Besonders deutlich wird dieser Bruch mit den Konventionen, wenn religiöse und sexuelle Bilder in einer Metapher miteinander in Verbindung gebracht werden. Die zweite Teilfrage, warum das Gedicht wohl posthum erschienen sei, zielt auf diesen Transfer des deklarativen Wissens.

Dieses wesentliche Ergebnis der FALKO-Deutsch-Studie war, dass der Zusammenhang zwischen fachwissenschaftlichem und fachdidaktischem Wissen in Deutsch mit einer Korrelation von $\mathrm{r}=0,51$ zwar substanziell, im Vergleich zu allen anderen Fächern jedoch am geringsten war. Ein möglicher Grund hierfür könnte sein, dass das Schulfach Deutsch von jeher auf die Vermittlung von (Schlüssel-)Kompetenzen wie Lesen, Schreiben, Rechtschreiben, Interpretationsoder Gesprächskompetenzen ausgerichtet ist und weniger auf die Vermittlung deklarativer bzw. akademischer Wissensbestände. Dazu bedarf es - etwa bei der Entwicklung von Lese- und Schreibkompetenz - eher eines soliden kognitionspsy- 
chologischen Wissens zu Lese- und Schreibprozessen, wenngleich textlinguistisches Wissen ebenfalls zum relevanten Wissen gezählt werden muss.

Eine zentrale Tatsache, die sich auch mit diesen empirischen Daten deckt, ist, dass Schulfächer weit davon entfernt sind, ein einfaches Abbild der akademischen Fächer zu sein. Sie sind autonome Gebilde, die ihrer eigenen Logik folgen und eine eigene Kultur bilden. Dabei stützen sie sich - besonders das Fach Deutsch - auf jahrhundertealte Traditionen. »Ihre Inhalte sind alles andere als homogen: Sie setzen sich aus sozialen Praktiken, wissenschaftlichem (Experten-)Wissen und schulspezifischen Inhalten, die für das Lehren entwickelt wurden, zusammen. Diese sind immer auf die Bildung der Person ausgerichtet und dadurch begründet.« (Schneuwly 2009, S. 316). Deutsch als Unterrichtsfach, so kann theoretisch wie empirisch geschlossen werden, hat geringere Überschneidungen mit dem Germanistikstudium als viele andere Schulfächer mit ihren jeweiligen akademischen Disziplinen, was auch der Tatsache geschuldet sein könnte, dass die Germanistik im 19. Jahrhundert aus der Notwendigkeit geboren wurde, Lehrkräfte für den muttersprachlichen Unterricht zu qualifizieren (vgl. Abraham 2019, S. 6), während in anderen Fällen das akademische Fach zum Schulfach wurde und dessen basale Wissensbestände übernahm. Abraham spricht sogar von »der Fiktion einer (Teil-)Identität von akademischer Disziplin und Schulfach«(Abraham 2019, S. 10).

Eine Möglichkeit, das Lehramtsstudium kohärenter zu gestalten, besteht sicherlich in einer inhaltlichen Annäherung im Rahmen der universitären Lehrerbildung. Das kann von einzelnen, aufeinander bezogenen Seminaren bis hin zu einer systematischen Verzahnung reichen. Als Beispiel hierfür können die Goethe-Universität Frankfurt und die Universität Passau dienen, an denen Inhalte der Kinderund Jugendliteraturforschung nicht von den Fachdidaktiken, sondern von den Fachwissenschaften für die Lehramtsstudierenden angeboten werden, während sich die Fachdidaktik auf den Vermittlungsprozess konzentriert, etwa die Frage, wie literarische Kompetenzen mit Kinder- und Jugendliteratur aufgebaut werden können (vgl. hierzu auch Abraham 2019, S. 7). Eine andere Möglichkeit wäre die Frage danach, was das »relevante Hintergrundwissen« für Prozesse wie »Schreiben«, »Gespräche führen «, »Sprachbewusstsein« oder »Textverstehen « ist. Diese Zugangsweise kehrte allerdings das um, was traditionell als »Abbilddidaktik« (vgl. Schröder 2015, S. 3) verstanden wurde (nämlich die didaktische Reduktion der germanistischen Forschungsergebnisse für die Schule). Stattdessen bildeten die schulischen Kompetenzen den Ausgangspunkt für die Frage, welches germanistische Wissen für diese Lehr-/Lernprozesse erforderlich ist (vgl. dazu auch Rothstein 2019, S. 15f.). Während es im Hinblick auf die zu lehrenden Inhalte bei gutem Willen aller Beteiligten also noch vergleichsweise einfach wäre, verwandtschaftliche Beziehungen mit dem Ziel einer kohärenten Lehrerbildung zu pflegen, wird es zunehmend schwieriger, wenn es um die Forschung geht.

\subsection{Neue Beziehungen im Kontext deutschdidaktischer Forschung}

Spätestens der PISA-Schock im Jahr 2000 war ein deutliches Indiz für die Deutschdidaktik, dass trotz des lebhaften fachdidaktischen Diskurses über »guten Deutschunterricht« zu wenig Wissen über die effektive Vermittlung fachspezifischer Kompe- 
tenzen vorhanden war (vgl. z. B. Groeben/Hurrelmann 2006, S. 26f.). Da die Untersuchung der Lesekompetenz überwiegend in den Zuständigkeitsbereich des Faches Deutsch fiel, war das Fach neben der Mathematik und den Naturwissenschaften eines der ersten, für die Bildungsstandards vorlagen (vgl. KMK 2003, 2004, 2012). Seit 2000 ist das Fach mit der permanenten Frage konfrontiert, wie sich Schule und Unterricht verändern müssen, um eine (inter-)nationale Spitzenposition bei den Leistungen der Schüler/-innen zu erreichen.

Folgt man Poppers Verdikt, »that we are not students of some subject matter, but students of problems. And problems may cut right across the borders of any subject matter or discipline « (Popper 1963, S. 88), und akzeptiert man, dass es - wie auch in der Qualitätsoffensive Lehrerbildung formuliert - die Aufgabe der Fachdidaktiken ist, einen intensiven Bezug zur schulischen Praxis im Sinne einer den Unterricht verändernden »eingreifende[n] Didaktik« (Schneuwly 2009, S. 316) herzustellen, dann stellt sich die Frage nach den richtigen, »befruchtenden« Beziehungen. Denn schon erste Schritte in die empirische Bildungsforschung führen dem oder der traditionell (also im Fach) sozialisierten Fachdidaktiker/-in vor Augen, dass es mit der Berechnung von Korrelationen und Effektstärken nicht getan ist, wenn komplexere Fragestellungen beantwortet werden sollen (vgl. Lindl et al. 2020). Transdisziplinäre Kooperationen mit der allgemeinen Bildungswissenschaft und Expertinnen und Experten für Methoden der empirischen Bildungsforschung sind nötig, um komplexe Systeme wie »Unterricht« oder komplexe Konstrukte wie »Lesekompetenz«, »literarische Kompetenz « oder auch »Lehrerprofessionalität« angemessen erforschen zu können.

Nun könnte man meinen, dass die Fachdidaktik von der kleinen, nicht immer als echte »Wissenschaft« ernst genommenen Schwester der Fachwissenschaften dadurch zum wenig geschätzten Adoptivkind der Bildungswissenschaften wird. Während dieses Bild nach der PISA-Studie durchaus zutreffend war, wird inzwischen aus den Bildungswissenschaften heraus das Desiderat einer größeren fachlichen Breite erkannt. Ein großer Teil der empirischen Unterrichtsforschung wurde in Mathematik oder den Naturwissenschaften durchgeführt (Praetorius 2018) und anschließend oft für allgemeingültig erklärt. Eine Metastudie von Seidel/Shavelson (2007) zeigt jedoch, dass Lernen gerade dann besonders effektiv ist, wenn der Unterricht domänenspezifische Lernprozesse fokussiert. Inzwischen ist in der Bildungswissenschaft unbestritten, dass zentrale Konstrukte, wie etwa die »kognitive Aktivierung «, vom Fach her gedacht und ausdifferenziert werden müssen (Klieme/Rakoczy 2008; Terhart 2006; Klieme/Leutner 2006; Feindt/Meyer 2010; Blömeke et al. 2015). Auch Schneuwly (2009, S. 323) konstatiert selbstbewusst: »Fachdidaktik steht im Zentrum der Unterrichtsforschung, da, wo es um das Wesentliche geht: Was wie unterrichtet wird und warum - aus der Perspektive der Lehrpersonen, aus der Schülerperspektive und aus Schulfach- und allgemeiner Schulperspektive.«

Forschungskooperationen zwischen der allgemeinen Bildungsforschung und den unterschiedlichen Fachdidaktiken ergeben sich von daher aus dem gemeinsamen Ziel, die Frage nach den Kriterien für qualitätsvollen Unterricht zu lösen. Dies macht einen transdisziplinären Ansatz unumgänglich. Mittelstraß zufolge handelt es sich bei »Transdisziplinarität« um mehr als um eine interdisziplinäre »Zusammenarbeit auf Zeit« (Mittelstraß 2005, S. 19). Unter »Transdisziplinarität« versteht er 
ein »Forschungs- und Wissenschaftsprinzip«, das jeweils dort greift, »wo eine allein fachliche oder disziplinäre Definition von Problemlagen und Problemlösungen nicht möglich ist «, also bei außerwissenschaftlichen, gesellschaftlichen Problemstellungen (Mittelstraß 2005, S. 21; Hervorh. im Orig.). Ein wesentliches Merkmal transdisziplinärer Forschungsfelder ist, dass es durch eine solche »Kooperation zu einer andauernden, die fachlichen und disziplinären Orientierungen selbst verändernden wissenschaftssystematischen Ordnung « kommt (Mittelstraß 2005, S. 19). Das bedeutet also, dass die einzelnen Disziplinen nicht bleiben, »was sie waren«, sondern »zumindest [...] ihre methodischen und theoretischen Perspektiven « ändern (Mittelstraß 2005, S. 23). Dieser Transformationsprozess lässt sich in den empirisch forschenden Fachdidaktiken deutlich ablesen. Sie erforschen - etwa im Bereich der Unterrichts- und Professionsforschung - sich gesellschaftlich ergebende Probleme und müssen für diese Forschung diejenigen Methoden verwenden, die sich hierzu eignen.

Dies heißt jedoch nicht, dass die Fachdidaktiken dadurch ihre fachliche Bindung verlieren. Diese ist es ja gerade, die den »Mehrwert« im Kontext der Bildungswissenschaften darstellt: »Disziplinäre Kompetenzen bleiben also die wesentliche Voraussetzung für transdisziplinär definierte Aufgaben, aber sie allein reichen nicht mehr aus, um Forschungsaufgaben, die aus den klassischen Fächern und Disziplinen herauswachsen, erfolgreich zu bearbeiten.« (Mittelstraß 2005, S. 20 f.).

Gerade im Kontext der Bildungswissenschaften ist das hervorstechende Merkmal des bzw. der Deutschdidaktiker/-in ihre germanistische Verwandtschaft. Empirisch forschende Deutschdidaktiker/-innen greifen dabei auf diejenigen fachlichen Forschungsergebnisse zurück, die für die Lösung ihrer transdisziplinären Forschungsfragen ergiebig erscheinen. Sie forschen dabei selbst jedoch meist nicht im fachlichen Kontext und verwenden nicht vorrangig fachwissenschaftliche Methodologie.

\section{Deutschdidaktische Forschung exemplarisch}

Im Folgenden sollen zwei exemplarische deutschdidaktische Forschungsprojekte vorgestellt werden, die prototypisch für einen transdiziplinären Forschungsansatz sind. Es sind die Projekte LUK (Literarästhetische Urteilskompetenz) und FALKE (Fachspezifische Lehrerkompetenzen im Erklären).

\subsection{Fallbeispiel 1: Das Projekt »Literarästhetische Urteilskompetenz«(LUK)}

Eine zentrale gesellschaftliche Aufgabe, die in der Folge des PISA-Schocks nach einer transdisziplinären Lösung verlangt, ist die Kompetenzforschung. Eine Offensive in diesem Bereich stellte das von dem Bildungsforscher Eckhard Klieme und dem Lehr-Lern-Psychologen Detlev Leutner eingerichtete DFG-Schwerpunktprogramm »Kompetenzmodelle zur Erfassung individueller Lernergebnisse und zur Bilanzierung von Bildungsprozessen « dar (vgl. Klieme/Leutner 2006). Innerhalb dieses Schwerpunktprogramms forschten zwischen 2007 und 201430 interdisziplinäre Projekte daran, »die kognitionspsychologischen und fachdidaktischen Grundlagen von Kompetenzen besser zu verstehen « und Instrumente zur Kompetenzmessung zu 
entwickeln, »um so langfristig die Vermittlung von Kompetenzen als zentrales Ziel schulischer und beruflicher Bildung zu unterstützen« (DIPF 2019). Erklärtes Ziel des Schwerpunktprogramms war es, »Expertinnen und Experten aus der Psychologie, der Erziehungswissenschaft und den Fachdidaktiken zusammen[zubringen] « (DIPF 2019). Die Projekte stammten aus »den Domänen Mathematik, Naturwissenschaft, Sprache/Lesen, Lehrerkompetenzen und fächerübergreifende Kompetenzen « (Fleischer et al. 2013, S. 6). Sie befassten sich u.a. mit physikalischen, naturwissenschaftlichen, umweltspezifischen, gesundheitsbezogenen sowie geographischen Fähigkeitsbündeln.

Das hier vorgestellte Teilprojekt »Literarästhetische Urteilskompetenz« (LUK) stellte mit dem Thema »Literarisches Textverstehen« das wohl exotischste Projekt dieses Schwerpunktprogramms dar, da es sich auf ein dezidiert geisteswissenschaftliches Kompetenzfeld bezog. Die Antragsteller/-innen entstammten drei Disziplinen: der Didaktik der deutschen Sprache und Literatur (Volker Frederking/Christel Meier), der empirischen Bildungsforschung (Petra Stanat/Thorsten Roick) und der pädagogischen Psychologie (Oliver Dickhäuser). Begünstigt wurde diese Kooperation durch die Bündelung der mit der Lehrer/-innenbildung befassten Disziplinen am Nürnberger Campus, wo sich neben empirischer Bildungsforschung, schulbezogener Pädagogik und Psychologie auch das »Department Fachdidaktiken« befindet.

Ziel des Projekts war die theoretische Modellierung und empirische Überprüfung eines Kompetenzmodells des literarischen Textverstehens.

Die erste Aufgabe auf fachdidaktischer Seite bestand dabei in der Erarbeitung eines literaturtheoretisch fundierten Kompetenzmodells. Diese »disziplinäre « Phase (Frederking/Henschel 2016, S. 81) wurde im Projekt LUK von Fachdidaktiker/-innen bestritten, die zugleich promovierte Literaturwissenschaftler/-innen sind. Zunächst musste eine literaturtheoretische Grundlage für das Kompetenzmodell gefunden werden, die die differenzierte Beschreibung von Teilfähigkeiten erlaubt, anschlussfähig an geltende Bildungsstandards ist und zudem eine Überprüfung von Teilfähigkeiten des literarischen Textverstehens ermöglicht. Außerdem sollte eine gewisse Kompatibilität mit kognitionspsychologischen Textverstehenstheorien vorhanden sein, da diese für die Modellierung von Lesekompetenz heute die Hauptreferenz darstellen. Für das literarische Verstehen bieten kognitionspsychologische Theorien allerdings keine hinreichende Theoriegrundlage, da darin unter anderem Text-Oberflächenstrukturen und rhetorischen Strategien zu wenig Aufmerksamkeit gezollt wird. Dies betonte 1994 auch Walter Kintsch und regte eine entsprechende Kooperation von Kognitionspsychologie und Literaturwissenschaft an: »bisher vernachlässigte Repräsentationen und die damit verbundenen Enkodierungsstrategien « müssten »analysiert, beschrieben und in ein solches Modell einbezogen werden « (Kintsch 1994, S. 41). Welche Strategien für literarisches Textverstehen relevant sind, sei dabei ein domänenspezifisches Problem: »Die Kognitionswissenschaft hat dazu nichts Eigenes beizutragen: sie beschäftigt sich allein mit der strategischen Konstruktion von Situationsmodellen; der sachliche Inhalt dieser Modelle jedoch und die Art der dazu benötigten Strategien sind bereichsspezifisch.« (Kintsch 1994, S. 50). Wo es bislang Versuche einer literaturtheoretischen Erweiterung kognitionspsychologischer Textverstehensmodelle gibt, stammen diese vor allem aus der empirischen Literaturwissenschaft (vgl. z. B. Vipond/Hunt 1984, S. 267 im Rückgriff auf Rosenblatt) 
und der Fachdidaktik (vgl. z. B. Rosebrock/Nix 2008, S. 119, die u.a. auf Schmidt rekurrieren, oder Grzesik 1990, S. 82, der sich auf Ingarden stützt). Gerade der Fachdidaktik kommt hier als Bindeglied zwischen den Disziplinen eine wichtige Vermittlungsrolle zu.

Als ergiebige literaturtheoretische Erweiterung kognitionspsychologischer Textverstehensmodelle fiel die Wahl im Projekt LUK auf Umberto Ecos literatursemiotischen Ansatz, der in der Literaturdidaktik bereits seit den 70er-Jahren intensiv rezipiert wird. Angesichts der eher marginalen Stellung, die Eco z. B. in germanistischen Überblickswerken zur Literaturtheorie innehat, mag das überraschen, zumal er dort unter den verschiedensten Schlagwörtern gehandelt wird: unter »Strukturalismus« (Köppe/Winko 2008, S. 47, 50f.) ebenso wie unter »Posthermeneutik « (Leiteritz 2004, S. 150-152). Gerade dass es Eco gelingt, »strukturale Textanalyse mit einer Theorie des Lesers auf Grundlage semiotischer und hermeneutischer Positionen« zu verknüpfen (Leiteritz 2004, S. 150), macht jedoch sein enormes Potenzial für die Literaturdidaktik aus. Ecos Vorstellungen $\mathrm{zu} »$ Grenzen der Interpretation« (Eco 1992) ermöglichen für die Modellierung von Textverstehenskompetenzen zudem, was über radikalkonstruktivistische oder poststrukturalistische Ansätze nicht möglich wäre: das Setzen von Normen als Beurteilungsgrundlage für Interpretationen. Zwar betont auch Eco, dass es die eine richtige Interpretation nicht geben kann, auf der Grundlage der »intentio operis « hält er - anders als auf Grundlage der »intentio lectoris« oder gar der »intentio auctoris« - die »Falsifizierung der Fehlinterpretationen« jedoch für möglich (vgl. Eco 1992, S. 51, 158 und zu den Intentionstypen S. 35-39; Hervorh. im Orig.). Für die Überprüfung von Kompetenzen in konkreten Aufgaben ist dies entscheidend (vgl. Frederking/Henschel 2016, S. 78f.).

Dabei ist Ecos Ansatz in vielerlei Hinsicht kompatibel mit grundlegenden kognitionspsychologischen Theorieannahmen (vgl. hierzu ausführlich Meier i. Vorb.). Beide Konzeptionen gehen z. B. im Sinne einer Kommunikationssituation von einer Interaktion zwischen Text und Leser aus (vgl. Eco 1973, S. 65; van Dijk/Kintsch 1983), wobei das Vorwissen und die Kompetenzen des Lesers von zentraler Bedeutung sind (vgl. Eco 1992, S. 187; Kintsch 1988). Im Zentrum beider Annahmen steht zudem das Herstellen von Textkohärenz. Vermutungen des Lesers müssen sich Eco zufolge stets »an der Kongruenz des Textes bewähren« (Eco 1992, S. 49). In kognitionspsychologischen Modellen wird die gesamte Sinnkonstruktion beim Lesen über das Herstellen lokaler und globaler Kohärenzen bis hin zu einem abstrahierten Situationsmodell konzipiert (vgl. van Dijk/Kintsch 1983). Übereinstimmungen gibt es auch bezüglich der Beibehaltung einer Intentionalitätsvorstellung. Diese wird in kognitionspsychologischen Theorien (oft recht autorintentionalistisch) auf der Ebene des vom Leser zu konstruierenden »pragmatischen Modells« angesetzt (vgl. Schnotz/Dutke 2004, S. 87). Eco löst das Problem der Intentionalität einerseits durch seine in den Text verlegten anthropomorphisierenden Konzepte der »intentio operis« bzw. der »Textstrategie« (vgl. z. B. Schalk 2000, S. 151, 164) und andererseits durch die Idee der Konstruktion eines »Modell-Autors« durch den Leser (Eco 1987a, S. 76f.). Seine Vorstellungen von Verstehensprozessen zeigen zudem Übereinstimmungen mit kognitionspsychologischen Ideen zu Inferenzen und Elaborationen. Eco setzt als »typisches Verfahren« literarischer Lektüren z. B. bei der Vereindeutigung mehrdeutiger Stellen die »Abduktion« an. Während der Lektüre aufgestellte Hypo- 
thesen werden dabei durch den Kontext bestätigt oder falsifiziert (vgl. Eco 1992, S. 295, 144). Diese Beschreibung ähnelt kognitionspsychologischen Beschreibungen der Sinnkonstruktion z. B. in Kintschs bekanntem »Construction-IntegrationModell «. Auch Kintsch zufolge werden im Leseprozess verschiedene Propositionen miteinander sowie innerhalb ihres Kontexts abgeglichen, wobei unzutreffende Inferenzen per Falsifikation ausgeschlossen werden (vgl. Kintsch 1988, S. 166-169). Ecos ausdifferenzierte Abduktionstypen leisten jedoch darüber hinaus, was allein auf den Online-Prozess (vgl. Zwaan 1993, S. 4) und primär auf die Erstellung eines Situationsmodells durch den Leser bezogene Inferenz- und Elaborationstypen der Kognitionspsychologie (vgl. z. B. Magliano et al. 1996) schuldig bleiben. Denn Ecos Schlusstypen sind auch für Post-Leseprozesse und Mehrfachlektüren (vgl. Eco 1973, S. 81 f.; Eco 1994, S. 41) geeignet und beziehen sich viel stärker auf semiotische Aspekte, die in kognitionspsychologischen Modellen höchstens angedeutet, aber nicht ausgeführt werden (vgl. van Dijk/Kintsch 1983, S. 17 f. zur rhetorischen und stilistischen Strategie).

Ein wichtiger Ansatzpunkt für die Modellierung von Teilfähigkeiten literarischen Textverstehens ist daher Ecos Unterscheidung zwischen »semantischer (»semiosischer«) und »kritischer« (»semiotischer«) Lektüre (vgl. Eco 1992, S. 43, Hervorh. i. Orig.). Diese Unterscheidung wurde Grundlage für die Modellierung der beiden zentralen Teilkompetenzen im Projekt LUK: für eine inhaltlich-semantische Teilfähigkeit, bei der es um die Herstellung eines kohärenten Textsinns geht, und eine nach Eco benannte formbezogen-»idiolektale« Kompetenzdimension des literarischen Textverstehens. Eco bezeichnet als »Idiolekt«»[d]ie Regel, die allen Abweichungen auf allen Ebenen eines Kunstwerks zugrunde liegt« (Eco 1987b, S. 362). Unter »idiolektaler literarästhetischer Urteilskompetenz« wurde im Projekt LUK daher die semiotische Fähigkeit gefasst, »zu erklären [...], aufgrund welcher Strukturmerkmale der Text diese (oder andere) semantischen Interpretationen hervorbringen kann« (Eco 1992, S. 43).

Ein Aufgabenbeispiel zur idiolektalen Dimension literarischen Textverstehens soll verdeutlichen, wie sich diese Orientierung an Eco in der Formulierung von Aufgaben niederschlug. So wurde z. B. zu einem Ausschnitt aus dem Jugendroman Tochterliebe von Rosemarie von Schach aus dem Jahr 1988 (Schach 1994, S. 5 f.) folgende Aufgabe gestellt:

In den Zeilen 13-14 heißt es »> Ja<, hört Jessica sich sagen«. Warum wurde Ihrer Meinung nach diese Formulierung gewählt und nicht » Ja<, sagt Jessica $\ll$ ?

Dieses offene Format erfordert zunächst, was Eco als »kreative Abduktion« bezeichnet. Bei diesem anspruchsvollen Schlussverfahren muss die Regel für eine Erklärung vom Leser »ex novo erfunden werden« (Eco 1985, S. 71; Hervorh. im Orig.). Das Aufstellen solcher Hypothesen zu sprachlichen Formulierungsvarianten oder Auffälligkeiten zeichnet sich durch einen hohen Abstraktionsgrad, einen hohen Grad an Interpretationsleistung und die Integration mehrerer Textinformationen aus und fällt Jugendlichen daher besonders schwer (vgl. Meier et al. 2013, S. 116f.). Des Weiteren zeigt die Formulierung »Warum wurde [...] diese Formulierung gewählt«, dass von der Intentionalität der Sprachwahl durch eine - hier nicht näher benannte Intentionsinstanz ausgegangen wird. Dies zeigt Affinitäten zu Ecos Konzeption des 
»Modell-Autors «. Der in der Aufgabe enthaltene Vergleich der fokussierten Formulierung mit einer Alternative entspricht Ecos »semiotische[m] Kommutationstest « zum Aufdecken von »Kontextsolidarität« (Eco 1987b, S. 360f.) und dient der Vereinfachung der Aufgabe. Geschlossene Aufgaben zur Funktion sprachlicher Formulierungen werden zudem häufig durch folgende Aufforderung eingeleitet: »Kreuzen Sie bitte an, welche Eindrücke die Textstelle beim Leser auslösen soll und welche nicht «. Auf Eco gehen hier die Betonung der »intentio operis« und die Vorstellung eines »Modell-Lesers« zurück (vgl. Eco 1987a, S. 63). Solchen Aufforderungen folgen daraufhin in der Regel mehrere plausible Antworten im Sinne einer Offenheit des Kunstwerks (vgl. Eco 1973) und nicht plausible, d.h. nicht mit der Textintention in Einklang zu bringende Lösungsvorschläge, die deduktiv überprüft und dann gegebenenfalls mittels Falsifikation ausgeschlossen werden.

Die beiden Kernkompetenzen des semantischen und des idiolektalen Textverstehens wurden im weiteren Projektverlauf um drei zusätzliche Kompetenzdimensionen ergänzt, die ebenfalls mit Ecos Semiotik im Einklang stehen: die »aufmerksame Sprachwahrnehmung «, das »literaturspezifische Fachwissen« und die Fähigkeit, textseitig intendierte Emotionen zu erfassen (vgl. Meier et al. 2017).

In dieser »disziplinäre[n] « Phase (Frederking/Henschel 2016, S. 81) des Projekts war die Verwandtschaft zwischen Literaturwissenschaft und Literaturdidaktik sicher besonders groß, zumal die beteiligten Didaktiker/-innen beide Disziplinen in sich vereinten. Dennoch ist das fachdidaktische Interesse im Zugriff auf die fachwissenschaftlichen Gegenstände hierbei prägend. Denn die Fragestellung dieser Phase lautete nicht abbilddidaktisch $»$ Welches ist die >richtige< Literaturtheorie, nach der in Zukunft auch Schüler/-innen zu unterrichten sind?«, sondern: »Welche Literaturtheorie stellt für schulische Belange einen angemessenen Referenzrahmen dar und ist anschlussfähig für andere interdisziplinäre Erklärungsmuster z. B. im Hinblick auf kognitive Prozesse? «

In den folgenden Phasen des Projekts nahm die Kooperation mit der empirischen Bildungsforschung und pädagogischen Psychologie deutlich zu und hatte drei Schwerpunkte: die Entwicklung von Fragestellungen und Hypothesen, die Aufgabenkonstruktion und die Interpretation der Befunde im Rahmen gemeinsamer Publikationen.

Folgende Forschungsfragen standen im Zentrum des Projekts: 1. Ist literarisches Textverstehen von Sachtextverstehen abgrenzbar? 2. Lässt sich die angenommene mehrdimensionale Kompetenzstruktur des theoretisch hergeleiteten Kompetenzmodells empirisch stützen? Hinzu kamen viele weitere Fragestellungen im Zuge der Validierung des Kompetenzmodells: zu Geschlechter- oder Schulformeffekten ebenso wie zu Zusammenhängen mit motivationalen Konstrukten oder allgemeinsprachlichen Fähigkeiten wie Sprachbewusstheit oder Wortschatzleistung. Diese Fragestellungen, die auch die Grenzen von Sprach- und Literaturdidaktik überschritten, wurden stets transdisziplinär theoretisch fundiert: fachdidaktische, kognitionspsychologische und erziehungswissenschaftliche Theorien und Studien wurden als Grundlage für Hypothesen zusammengeführt. Natürlich waren dabei auch fachliche Differenzen zu überwinden, und bisweilen waren intensive Recherchen nötig, um eingeführte fachdidaktische Konstrukte im internationalen erziehungswissenschaftlichen oder lernpsychologischen Diskurs zu verorten. 
Ein zweites Kooperationsfeld war die Aufgabenkonstruktion. Zwar lag diese primär in deutschdidaktischer Hand, durch ein mehrstufiges Verfahren der Aufgabenentwicklung war die Interaktion der beiden Disziplinen jedoch stark ausgeprägt. Gerade zu Projektbeginn wurden Aufgaben ausführlich gemeinsam besprochen und Vor- und Nachteile möglicher Formate diskutiert. In gemeinsamen Sitzungen wurden die in »Cognitive Laboratories « und Pilotierungsstudien erprobten Items intensiv überarbeitet. Während die Vertreter/-innen der empirischen Bildungsforschung Aufgaben primär nach statistischen Kriterien beurteilten (also z. B. nach einer ausgewogenen Schwierigkeitsverteilung oder der Trennschärfe), beurteilten die Fachdidaktiker/-innen diese v.a. im Hinblick auf ihre fachliche und fachdidaktische Angemessenheit. Dabei zeigten sich v.a. anfangs auch Differenzen zwischen den Disziplinen z. B. im Hinblick auf die Mehrdeutigkeit literarischer Texte, den Eigenwert literarästhetischer Sprachreflexion, den Anspruch an die Komplexität von Aufgaben oder die Möglichkeit, Aufgaben eindeutig als richtig oder falsch zu bewerten. Im interdisziplinären Diskurs galt es, Kompromisse zu finden. So bildete sich z. B. eine zunehmend abgestufte Beurteilung der Aufgaben heraus, und auch im Hinblick auf die Deutungsoffenheit der Texte wurden gemeinsame Lösungen gefunden. Dazu gehörten unter anderem die oben beschriebenen Wahlaufgaben mit mehreren korrekten Antworten und das Beibehalten auswertungsintensiver offener Aufgabenformate auf Wunsch der Didaktik.

Eine noch intensivere Kooperation war schließlich bezüglich der Interpretation und Publikation der Daten nötig. Dabei wurden für beide Disziplinen äußerst interessante, gemeinsame Ergebnisse und interdisziplinäre Synthesen präsentiert (vgl. u.a. Meier et al. 2017). Hinzu kamen eigenständige Qualifikationsarbeiten aus dem Projektkontext: die Dissertationen von Lydia Steinhauer (2010) zu »Involviertem Lesen« und Sofie Henschel (2013) zu »Effekten motivationaler und affektiver Merkmale auf das Verstehen von literarischen und faktualen Texten« sowie die Habilitationsschrift von Christel Meier zu »Literarästhetischer Sprachreflexion« (Meier i. Vorb.). In diesen Arbeiten zeigt sich am deutlichsten die »methodische Transdisziplinarität« in Mittelstraß' Sinn, da die Autorinnen hier Methoden und Konzepte des jeweils »fremden « Wissenschaftsparadigmas anwendeten und adaptierten.

Einige zentrale Ergebnisse des Projekts sollen an dieser Stelle schlaglichtartig auch im Hinblick auf ihre Relevanz für Curricula und Förderansätze zusammengefasst werden.

Ein erstes bedeutsames Ergebnis der LUK-Studie ist die empirische Trennbarkeit von »Sachtextverstehen« und »Literarischem Textverstehen« (vgl. Meier et al. 2017, S. 56) entgegen der in der Lesedidaktik verbreiteten Meinung, dass es »nicht sinnvoll« sei, »zwischen dem Lesen literarischer und nicht-literarischer Texte« zu unterscheiden (Groeben 2006, S. 12). Mit einer latenten Korrelation von $r=0,76$ $(p<0,01 ; N=960)$ hängen die beiden Kompetenzen bei Schüler/-innen der 10. Jahrgangsstufe zwar substanziell, aber nicht zu hoch zusammen (vgl. zur Höhe solcher Korrelationen Artelt/Schlagmüller 2004, S. 171). Die LUK-Studie stützt damit Ergebnisse von Reanalysen der PISA-2000-Daten von Artelt und Schlagmüller, in denen die Textverstehensleistungen bei literarischen und nicht-literarischen Texten zu r=0,79 latent korrelierten (vgl. Artelt/Schlagmüller 2004, S. 179). Dass es sich auch in qualitativer Hinsicht um verschiedene Kompetenzen handelt, zeigt zum 
Beispiel die Tatsache, dass das im Projekt LUK über eine Fragebogenskala erhobene Konstrukt der emotionalen Fantasieempathie nur ein signifikanter Prädiktor für das literarische Textverstehen, nicht aber für das Sachtextverstehen ist (vgl. Henschel/Roick 2013, S. 109 f.; Henschel 2013). LUK löst damit ein, was Artelt und Schlagmüller bereits 2004 im Hinblick auf eine literaturspezifische Lesekompetenz forderten, nämlich verstärkte Forschungsanstrengungen, »um zu einem differenzierteren Verständnis dieser Teilkompetenz zu gelangen und das Wissen über die spezifischen Verstehensanforderungen für die Förderung dieser Kompetenz nutzbar zu machen.« (Artelt/Schlagmüller 2004, S. 189). Dieser Befund ist von erheblicher Relevanz für die Formulierung von Bildungsstandards und Curricula. Darin sollten differenziertere Kompetenzziele für literarisches Verstehen und Sachtextverstehen ausgewiesen werden, als dies bislang der Fall ist. So wird z. B. im Primarbereich derzeit nicht auf semiotische Aspekte des literarischen Verstehens Bezug genommen (vgl. KMK 2004). Damit werden Chancen vergeben, spezifische Textverstehenskompetenzen von Anfang an anzubahnen. Die aktuelle IGLU-Studie zeigt Defizite deutscher Grundschüler/-innen beim Prüfen und Bewerten des »Sprachgebrauchs« und bei »Aufgaben, die sich auf Stil und Struktur von Texten beziehen, sowie bei Aufgaben, in denen nach Perspektive oder Absicht des Textes beziehungsweise des Autors gefragt wird « (Bos et al. 2017, S. 17, 25). Deutsche Grundschüler/-innen schneiden im internationalen Vergleich also genau bei den Fähigkeiten signifikant unterdurchschnittlich ab, die typischerweise an literarischen Texten erworben werden. Da Kinder durchaus in der Lage sind, literarische Texte auch als solche zu rezipieren, wie eine an LUK angelagerte Lautdenkstudie mit Grundschüler/-innen zeigte (vgl. Stiller 2017), sollten diese Kompetenzen auch von Anfang an angebahnt werden.

Wichtig ist in diesem Kontext auch, dass es nicht egal ist, was Jugendliche in ihrer Freizeit lesen. Denn diejenigen, die vor allem literarische Texte rezipieren, verstehen beides besser: Sachtexte und literarische Texte. Beim Erfassen der Textstrategie literarischer Texte haben Jugendliche, die in ihrer Freizeit Sachtexte präferieren, sogar kleine signifikante Nachteile (Meier 2020b, S. 142). Leseförderung sollte daher nicht nur auf die Quantität des Gelesenen abzielen, sondern vor allem versuchen, Kinder und Jugendliche an die Lektüre literarischer Ganzschriften heranzuführen.

Weitere zentrale Befunde der LUK-Studie beziehen sich auf die Frage nach der Unterscheidbarkeit einer auf die Semantik und einer auf die Form bezogenen Teilkompetenz des literarischen Textverstehens. Diese Trennung wird in der Literaturwissenschaft und -didaktik häufig abgelehnt. Die LUK-Studie setzte diese Unterscheidung nach Eco als semantische und semiotisch-idiolektale Lektüre daher auch ein Stück weit als Heuristik an und kann nun interessante Einblicke in den Zusammenhang zwischen beiden Kompetenzfacetten bei Jugendlichen geben. So zeigten die Untersuchungen mit Schüler/-innen der 9. Jahrgangsstufe, dass semantische und idiolektale Textverstehenskompetenz bei Mittelschüler/-innen am wenigsten zusammenhängen $(\mathrm{r}=0,54 ; p<0,05 ; N=1168)$, bei Realschüler/-innen etwas mehr ( $\mathrm{r}=0,77$; $p<0,05 ; N=1168)$ und bei Gymnasiastinnen und Gymnasiasten ( $\mathrm{r}=0,84 ; p<0,05$; $N=1168$ ) am deutlichsten (vgl. Roick et al. 2013, S. 80). Dieser Zusammenhang steigt bei Lernenden aus 10. Klassen in Gymnasien und Realschulen sogar noch etwas weiter an (r=0,89; $p<0,05 ; N=964$, vgl. Meier et al. 2017, S. 66). Während in 
der Mittelschule dem Literaturunterricht insgesamt wenig Bedeutung beigemessen wird (vgl. Pieper et al. 2004) und die Jugendlichen dort bei formbezogenen Aufgaben wesentlich schlechter abschnitten als bei inhaltsbezogenen (vgl. Roick et al. 2013, S. 80), ist die idiolektale Teilkompetenz in Realschule und Gymnasium stärker entwickelt. Bei sehr fortgeschrittenen Lernenden gehen semantische und idiolektale Textverstehenskompetenz zunehmend Hand in Hand. Auch hier zeigt die LUK-Studie also durchaus institutionellen Handlungsbedarf auf. Verzichtet man in manchen Bildungsgängen auf eine Auseinandersetzung mit sprachreflexiven Aspekten des Textverstehens, ist dies letztlich auch problematisch im Hinblick auf eine souveräne, emanzipierte Sprachbeherrschung ganzer gesellschaftlicher Gruppen (vgl. de la Combe 2007).

Ein weiterer relevanter Befund betrifft die Rolle deklarativ-konzeptuellen Fachwissens für das literarische Textverstehen. Hier ist in den letzten Jahrzehnten in der Deutschdidaktik häufig Kritik am Umgang mit klassischen Fachwissensinhalten geübt worden. Etliche Kritiker betonten sogar, dass Fachwissen literarisches Verstehen behindere (vgl. zusammenfassend Spinner 2012). Winkler attestiert der Literaturdidaktik ein chronisch gespaltenes Verhältnis zum Fachwissen (vgl. Winkler 2016, S. 61). Dass konzeptuell-deklaratives Fachwissen zum Beispiel zu Gattungsmerkmalen, Tropen, rhetorischen Figuren etc. durchaus substanzielle positive Zusammenhänge mit weiteren inhalts- und formbezogenen Facetten des literarischen Textverstehens hat, ist für die Literaturdidaktik daher durchaus ein wichtiger Befund. In einem Modell, das auf einer ersten Ebene Sachtextverstehen und literarisches Verstehen trennt und dann verschiedene Facetten literarischen Textverstehens ausdifferenziert, korreliert Fachwissen mit dem semantischen und idiolektal-formbezogenen Textverstehen sowie mit literarästhetischer Sprachwahrnehmung gleichermaßen zu r=0,72 ( $p<0,01 ; N=964$; vgl. Meier et al. 2017, S. 66). Auch wenn es sich bei literaturspezifischem Fachwissen um eine gut abtrennbare Kompetenzfacette handelt, so zeigen diese Werte doch, dass Jugendliche mit profunden Fachwissenskenntnissen in der Regel auch diejenigen sind, die literarische Texte besser verstehen oder sprachliche Auffälligkeiten sicherer identifizieren. Für die Gestaltung von Curricula und die Konzeption der Abituraufgaben ist auch dies ein bedeutendes Ergebnis, das dem Trend, Fachwissensinhalte im Deutschunterricht immer weiter zurückzudrängen und sogar in den Abiturprüfungen nicht mehr zum Gegenstand zu machen, entgegensteht.

Eine weitere Kompetenzfacette, die sich in den statistischen Analysen als eigenständig erwies, aber dennoch substanzielle Zusammenhänge mit den übrigen Kompetenzfacetten aufwies, ist das aufmerksame Wahrnehmen von Sprache in literarischen Texten. Diese Kompetenzfacette hängt bei Lernenden der 10. Jahrgangsstufe mit dem semantischen Textverstehen zu r=0,66; mit dem idiolektalen Textverstehen $\mathrm{zu} \mathrm{r}=0,68$ und mit dem literaturspezifischen Fachwissen zu r=0,72 zusammen $(p<0,01 ; N=964 ;$ vgl. Meier et al. 2017, S. 66). Dieser Aspekt des literarischen Verstehens, bei dem die meisten Prozessvorstellungen von literarischem Verstehen ansetzen (vgl. z. B. zum »Foregrounding « van Holt/Groeben 2005), wird in etlichen literaturdidaktischen Kompetenzmodellen als unterstes Niveau des Textverstehens modelliert (vgl. z. B. Boelmann et al. 2020, S. 17 f.). Wird eine Auffälligkeit nur entdeckt, aber nicht in ihrer Funktion beschrieben, handelt es sich solchen Kompetenzmodellen zufolge um defizitäres literarisches Verstehen. Problematisch ist 
daran, dass literarästhetische Sprachwahrnehmung so gar nicht als eigenständige Kompetenzfacette in den Blick gerät. Auch hier gibt es, wie die LUK-Studie zeigt, durchaus verschieden schwierige Anforderungen. So ist es z. B. anspruchsvoller, einen grammatisch oder syntaktisch bedingten Stilwechsel zu erkennen als ein auffälliges sprachliches Bild, und das Beschreiben stilistischer Auffälligkeiten in eigenen Worten ist schwieriger als das Herausschreiben von Auffälligkeiten (vgl. Meier i. Vorb.). Ein Niveaustufenmodell, das jede Art von Aufmerksamkeit für Sprache als unterstes Niveau ansetzt, wird dieser - auch im Hinblick auf die erforderlichen Prozesse - klar unterscheidbaren Kompetenz nicht gerecht. Konzipiert man diesen Aspekt aber als eigenständige Kompetenzfacette, kann auch die Aufmerksamkeit für Sprache als Förderaspekt mehr in den Blick geraten, als dies gegenwärtig der Fall ist. Diese Aufwertung der Begegnung mit der Sprachlichkeit von Texten sollte auch in den Curricula erfolgen. Dort ist sie - wohl auch aufgrund der Dominanz einer allgemeinen Lesedidaktik - bislang wenig vertreten.

Über die curricularen Implikationen der Trennbarkeit und Zusammenhänge von Teilkompetenzen hinaus bietet die LUK-Studie aber auch für die Förderung dieser Kompetenzen wichtige Ansatzpunkte. Durch das Inbezugsetzen der Kompetenzfacetten zu Faktoren wie Schulart, Geschlecht, Sprachhintergrund, Form- oder Inhaltsinteresse, Empathie etc. lassen sich differenziert Risikogruppen und Ansatzpunkte für die Förderung einzelner Teildimensionen ermitteln. Durch die Untersuchung von Zusammenhängen mit weiteren Kompetenzkonstrukten lassen sich Bereiche identifizieren, die für die Förderung des literarischen Textverstehens stärker fruchtbar gemacht werden sollten als bisher. So erwies sich z. B. im Hinblick auf alle Teilfacetten der literarästhetischen Sprachreflexion der Wortschatz als starker Prädiktor, während die Zusammenhänge mit Fähigkeiten im Bereich der allgemeinen prozeduralen Sprachbewusstheit eher gering ausfielen. Die Wortschatzleistung korrelierte in einem Strukturgleichungsmodell, das Geschlecht, Schulform und Sprachhintergrund als Kontrollvariablen einbezieht, mit dem Erfassen der Textstrategie latent $\mathrm{zu} \beta=0,62$; mit formspezifischem Fachwissen $\mathrm{zu} \beta=0,48$ und mit literarästhetischer Sprachwahrnehmung zu $\beta=0,28(p<0,01 ; N=964)$, während allgemeine prozedurale Sprachbewusstheit nur mit literarästhetischer Sprachwahrnehmung vergleichsweise gering latent korreliert $(\beta=0,20 ; p<0,05 ; N=964$; vgl. Meier 2020a, S. 228). Daher wäre eine intensivere Verknüpfung des literarischen Textverstehens mit Wortschatzarbeit in einem sprachreflexiven Literaturunterricht durchaus ein lohnendes Feld.

Zuletzt soll darauf hingewiesen werden, dass das im LUK-Modell entwickelte Testinstrument, das im Unterschied zu allen anderen verfügbaren Lesetests spezifisch literarische Teilfähigkeiten operationalisiert, überaus gewinnbringend in der Unterrichtsforschung eingesetzt werden kann, um Effekte literaturdidaktischer Lernarrangements differenziert zu untersuchen. Hier konnten bereits in zwei Studien interessante Ergebnisse zu zentralen deutschdidaktischen Konzeptionen herausgearbeitet werden: Henschel, Meier und Roick setzten den Test für einen Vergleich analytischer und handlungs- und produktionsorientierter Lernaufgaben ein, Frederking, Brüggemann und Albrecht benutzten ihn für die Evaluation offener und gelenkter Unterrichtsgespräche. Dabei zeigte sich, dass handlungs- und produktionsorientierte Lernaufgaben und offene Unterrichtsgespräche u.a. im Hinblick auf motivationale Aspekte deutliche Vorteile bieten, analytische Lernaufgaben und gelenkte Unter- 
richtsgespräche aber zu besseren Textverstehensleistungen im idiolektal-formbezogenen Bereich führten (vgl. Henschel et al. 2016, S. 17; Frederking et al. 2020, S. 303).

Dass Winkler und Schmidt in ihrem Band »Interdisziplinäre Forschung in der Deutschdidaktik « das LUK-Projekt als »vorwiegend wissenschaftsgeleitet « begreifen (Winkler/Schmidt 2016, S. 12) und nicht als gemeinsame Arbeit an einem »außerwissenschaftliche[n] Problem «, wie es Mittelstraß zufolge als Kennzeichen transdisziplinärer Forschung gelten kann (Mittelstraß 2005, S. 21), überrascht vor diesem Hintergrund etwas. Denn Kompetenzfacetten literarischen Verstehens werden im Projekt »Literarästhetische Urteilskompetenz« ja nicht zum Selbstzweck oder zur Optimierung psychometrischer Verfahren untersucht, sondern im Hinblick auf sinnvolle Kompetenzziele in Bildungsstandards und Curricula sowie im Hinblick auf lohnende Förderansätze im Deutschunterricht.

Die oben skizzierte enge Zusammenarbeit der beteiligten Disziplinen während der verschiedenen Projektphasen sollte zudem gezeigt haben, dass diese Kooperation weit über eine »Auxiliary Interdisciplinarity « nach Heckhausen (1972, S. 87-89) hinaus geht, der Winkler und Schmidt das LUK-Projekt zuordnen. Bei solch einem relativ geringen Integrationsgrad der fachlichen Perspektiven hätten empirische Bildungsforschung und Deutschdidaktik lediglich den Charakter von Hilfswissenschaften für die jeweils andere Disziplin, was Winkler und Schmidt noch nicht als Transdisziplinarität einstufen (vgl. Winkler/Schmidt 2016, S. 13). Natürlich bestand die Kooperation der Projektpartner nur »auf Zeit«, was auch nach Mittelstraß für Inter-, nicht für Transdisziplinarität spräche (vgl. Mittelstraß 2005, S. 19). Aufgrund des an LUK beobachtbaren nachhaltigen Wandels der »methodischen und theoretischen Perspektiven « (Mittelstraß 2005, S. 23) und der gemeinsamen Arbeit an einem außerwissenschaftlichen, gesellschaftlich relevanten Problem hat das Projekt unserer Ansicht nach jedoch ebenso exemplarischen Charakter für die Transdisziplinarität der Deutschdidaktik wie das nachfolgende Fallbeispiel.

\subsection{Fallbeispiel 2: FALKE}

FALKE (Fachspezifische Lehrkraftkompetenzen im Erklären) ist ein Teilprojekt des im Rahmen der Qualitätsoffensive Lehrerbildung des BMBF eingeworbenen Gesamtprojekts KOLEG (Kooperativ Lehrerbildung gestalten). Am Projekt FALKE partizipieren elf Fachdidaktiken an der Universität Regensburg. Geleitet wird das Projekt von Stefan Krauss (Mathematikdidaktik) und Anita Schilcher (Deutschdidaktik). Die methodische Beratung sowie das Datenmanagement im Projekt übernahm die Professur für Methoden der empirischen Bildungsforschung (Sven Hilbert und Alfred Lindl). In den FALKE-Folgeprojekten (FALKE 2 und FALKE-digital) vervollständigte Sven Hilbert die Projektleitung.

Das Projekt FALKE fußt auf dem Vorläuferprojekt FALKO (Fachspezifische Lehrerkompetenzen), das wiederum an die COACTIV-Studie anschließt. Damit steht es in der Tradition der Professionswissensforschung, die seit der ersten PISA-Studie (2000) einen enormen Aufschwung erlebt hat.

Ziel des FALKE-Projekts ist es, eine Facette dieses professionellen Wissens, nämlich die fachdidaktische Facette »Erklären« genauer zu untersuchen. Dabei stehen 
neben thematisch engeren und fachspezifischen Kriterien auch überfachliche prozedurale und performative Kompetenzen von Lehrkräften im Fokus, wie etwa die sprachliche Gestaltung von mündlichen Erklärungen sowie der Sprech- und Körperausdruck der Lehrkräfte. Dies führte in FALKE-1 zu einer interdisziplinären Zusammenarbeit mit der Linguistik und der Sprechwissenschaft, die hierfür ihre fachliche Expertise einbrachten und auf die entstandenen mündlichen Texte anwendeten (zu spezifischen inter- und transdisziplinären Aspekten vgl. Schilcher et al. 2021).

In FALKE wird das Konstrukt des Erklärens theoretisch aus der Perspektive verschiedener wissenschaftlicher Disziplinen beleuchtet, geschärft und empirisch untersucht. Dazu wurden pro Fach sechs kurze Erklärsituationen videografiert, die in einen für alle beteiligten Fachdidaktiken im Wesentlichen identischen online-basierten Fragebogen implementiert wurden. Die Qualität der gezeigten Erklärungen wurde anschließend von vier Statusgruppen (Schüler/-innen, Lehramtsstudierende, Lehrkräfte und Fachdidaktiker/-innen) beurteilt, wobei alle Studienteilnehmer/-innen zunächst eine Globalbeurteilung (Gesamtnote der didaktischen Qualität) abgaben und in einem zweiten Durchlauf dieselben Videos noch einmal mit Bezug auf Kriterien guten Erklärens wie beispielsweise Strukturiertheit oder Adressatenorientierung beurteilten. Im Zentrum stehen dabei Fragen wie: Welche Kriterien sind für »gutes Erklären« besonders zentral? Welche Gemeinsamkeiten, aber auch welche Unterschiede lassen sich dabei zwischen verschiedenen Unterrichtsfächern feststellen? Welche Fachspezifika zeigen sich im Vergleich mit den anderen Fächern?

Um diese Fragen beantworten zu können, einigte sich die Gruppe der elf Fachdidaktiken (jeweils bestehend aus Betreuer/-in und Doktorand/-in) auf allgemeine (vermutete) Qualitätsaspekte guten Erklärens wie etwa die Strukturiertheit, die Adressatenorientierung, die sprachliche Verständlichkeit sowie den Sprech- und Körperausdruck. Darüber hinaus konnte jedes Fach einen eigenen Aspekt guten Erklärens festlegen. Im Fach Deutsch wurde die »Erklärtiefe« als Konstrukt gewählt. Mit ihr sollte das Potential zur kognitiven Aktivierung einer Erklärung erfasst werden. Bei der Erklärtiefe kommt der disziplinäre Hintergrund zum Tragen. Denn um einen Inhalt vertieft in seinen Zusammenhängen erklären zu können, ist ein vertieftes fachliches Wissen erforderlich. Darüber hinaus rückt die disziplinäre Perspektive bei FALKE besonders bei der Wahl des zu erklärenden Inhalts ins Zentrum. Nach Befragungen von Seminarlehrkräften, die besonders problemhaltige Unterrichtssituationen schildern sollten, fiel die Wahl auf das Thema »schriftliches Argumentieren«. Die unterschiedlichen Inhalte der Erklärung entstammten alle diesem Themenkreis. Je zwei dreiminütige Video-Vignetten hatten den gleichen Inhalt: Die jeweilige Lehrkraft sollte erklären, warum folgende Argumentation aus einer Zeitschrift nicht gelungen war:

Ein Verbot [von Freundschaften zwischen Schülern und Lehrern auf Facebook] finde ich unsinnig, da man mit seinen Schülern ohnehin in irgendeiner Form in Kontakt steht. Und wer eben gern kumpelhaft ist, soll das doch bitte sein dürfen. Was ist so schlimm daran, wenn man ein entspanntes Verhältnis zu den Menschen hat, die einen täglich umgeben? (Ausschnitt aus www.fluter.de/likeden-lehrer). 
Während die eine »oberflächliche« und für den Deutschunterricht durchaus typische Erklärung die formal-strukturelle Schwäche des Arguments erklärte - die Begründung bezieht sich nicht auf die Behauptung, das Beispiel fehlt -, fokussierte die entsprechende tiefenstrukturelle Erklärung die fehlende funktional-inhaltliche Erklärung - also die Vernachlässigung der Tatsache, dass Lehrer-Schüler-Beziehungen institutionell geprägt sind und ein hierarchisches Verhältnis einschließen sowie das Fehlen eines Arguments, das die Unsinnigkeit des Verbots begründet. In einem weiteren Vignettenpaar erklärte die Lehrkraft die Redewendung »um etwas viel Aufhebens machen« einmal oberflächlich mit synonymen Ausdrücken (z. B. »um etwas viel Wind machen«) und einmal tiefenstrukturell im Hinblick auf die etymologischen Hintergründe, also die Entstehung der Redewendung im Kontext des mittelalterlichen Waffenkampfs (vgl. zu den Beispielen ausführlich Gaier i. Vorb.).

Bei der Auswertung der Befragung konnte in linearen hierarchischen Regressionsmodellen gezeigt werden, dass sich für die Statusgruppen unterschiedliche signifikante Prädiktoren für die globale Einschätzung der Erklärung ergeben: Während für Schüler/-innen nur der Sprech- und Körperausdruck einen signifikanten Einfluss auf die globale Bewertung der Erklärung hat, spielen für die Expertengruppe aus Studierenden, Lehrkräften und Fachdidaktiker/-innen die Strukturiertheit, die Adressatenorientierung sowie die Erklärtiefe eine entscheidende Rolle im Fach Deutsch. Der sprachlichen Gestaltung, so wie sie erfasst wurde, kommt in FALKE-D wie auch in den meisten Fächern dagegen keine Bedeutung zu. Gleiches gilt für das Kontrollkonstrukt der Persönlichkeitswirkung (vgl. Gaier/Schilcher i. Vorb.). Die Ergebnisse zeigen auch in dieser Studie, dass die spezifisch fachdidaktische Expertise von der »Expertengruppe « als besonders zentral wahrgenommen wird. FALKE-1 untersucht zunächst die wahrgenommene Qualität von Erklärungen, in weiteren Studien muss geklärt werden, welche der als wichtig wahrgenommenen Kriterien die Verstehensleistung der Schüler/-innen am stärksten beeinflussen. Die detaillierte Auswertung der Daten und die Fortführung der Untersuchungen in weiteren Teilstudien, wie sie in LUK erfolgte, steht FALKE noch bevor.

Die große Bedeutung der methodischen Transdisziplinarität wird jedoch auch bei FALKE besonders deutlich. Hier wurde in elf Fächern ein gemeinsames Design umgesetzt, um die Qualität von Unterrichtserklärungen zu untersuchen. Dies ermöglicht zum einen die breite Untersuchung von Kriterien für gute Erklärungen in den jeweiligen Fächern, liefert aufgrund des parallelen Vorgehens aber auch Befunde zu Ähnlichkeiten und Unterschieden zwischen den Fächern. In Meta-Analysen, die die in den einzelnen Fächern gewonnenen Erkenntnisse aufgreifen, können Verallgemeinerungen vorgenommen werden, die auf der Basis heterogener methodischer Ansätze nicht möglich wären. Auf diese Weise erfüllt FALKE eine grundlegende Forderung an die transdisziplinäre Forschung, nämlich einen Beitrag zu einer weiter gefassten Frage zu leisten, die nicht nur auf ein Thema oder Fach bezogen und darüber hinaus auch praxisrelevant ist. 


\section{Zur Angemessenheit des Verwandtschafts- und Beziehungsframings im Hinblick auf deutschdidaktische Kooperationen}

Doch welche Bedeutung hat diese neue Allianz der Fachdidaktik Deutsch mit empirischen Bildungswissenschaften? Familien- und Beziehungsmetaphern wie »Wahlverwandtschaften« haben hierfür durchaus Konjunktur. Iris Winkler und Frederike Schmidt wählten z. B. für ihren Band »Interdisziplinäre Forschung in der Deutschdidaktik« den Untertitel »>Fremde Schwestern< im Dialog« (Winkler/Schmidt 2016). Dabei griffen sie auf einen Beitrag von Ewald Terhart aus dem Jahr 2002 zurück, der mit dieser Metapher die Spannungen zwischen Allgemeiner Didaktik und empirischer Bildungsforschung umschrieb (Terhart 2002), und erweiterten sie darüber hinaus auf Fachdidaktik und Fachwissenschaft. Auf die Projekte FALKE und LUK trifft diese Metapher nur bedingt zu. Auch wenn manche Literaturwissenschaftler wie Boyken bedauern, »dass sich die Deutschdidaktik in ihren Erkenntnisinteressen und Untersuchungsmethoden von der Literaturwissenschaft entfernt hat « (Boyken 2016, S. 39), so ist die Klage über eine völlige Entfremdung der »Schwestern« doch unbegründet. Denn die Fachdidaktik Deutsch ist ja gerade durch ihre germanistische Fundierung definiert und nur aufgrund ihrer disziplinären Expertise ein gesuchter Partner in transdisziplinären Forschungsverbünden. Dass auch Fachwissenschaften bzw. fachwissenschaftliche Perspektiven in solchen Projekten einen zentralen Stellenwert haben, sollten die Fallbeispiele gezeigt haben.

Bei dem vorliegenden Heft wurde mit der Metapher der »Wahlverwandtschaften« ein anderes Bild für interdisziplinäre Beziehungen gewählt. Dass dieser Begriff die aktuellen Entwicklungen im Bereich der Deutschdidaktik treffender beschreibt als die Metapher der »fremden Schwestern«, darf mit einem kurzen Blick auf Goethes Roman allerdings bezweifelt werden. Denn in dieser Logik hätten Fachdidaktik und Fachwissenschaft wie Charlotte und Eduard lange Zeit einträchtig ihren germanistischen Landschaftspark bestellt, ehe das Auftreten des rationalen Empirikers Otto diese harmonische Verbindung zunichtemachte. Seine wahlverwandtschaftliche Anziehungskraft auf die ähnlich empirieaffine Charlotte wäre dann nicht nur der Anfang vom Ende der friedlichen Koexistenz von Germanistik und Deutschdidaktik, sondern auch das Signal für den Niedergang beider Disziplinen. Denn die neue Beziehung wird ja weder eingegangen noch fruchtbar, und die Frucht der alten Beziehung geht darüber ebenso zugrunde wie (Fachwissenschaftler?) Eduard. Derart pessimistisch stellt sich die Lage angesichts der durchaus fruchtbaren fachdidaktischen Forschungsverbünde und einer recht lebendigen Fachgermanistik ja keineswegs dar. Auch ohne zu betonen, dass die Besetzung der Ottilie in diesem Gedankenspiel schwerfällt, zeigt sich deutlich, dass dieses Wahlverwandtschafts-»Framing « nicht als Charakterisierung des Verhältnisses von Deutschdidaktik, Fachgermanistik und weiteren bildungswissenschaftlichen Bezugsdisziplinen passen mag. Unangemessen ist dabei nicht nur das Denken in sich ausschließenden Paarbeziehungen, das für transdisziplinäre Verbundforschung, in deren Kontexten fachdidaktische Forschung heute zunehmend praktiziert wird, unpassend ist, sondern auch die Emotionalisierung des Verhältnisses als »entfremdet«, »vertraut « oder gar »leidenschaftlich«. Geht man davon aus, dass die Fachdidaktik Deutsch ihre wissenschaftliche Existenzberechtigung primär aus der Lösung gesellschaftlich relevanter domänenspezifischer 
Bildungsprobleme bezieht, darf es weniger um wissenschaftliche Affinitäten gehen als um notwendige wissenschaftliche Kooperationen zur professionellen Lösung solcher Probleme.

Bleibt zu prüfen, ob die Metapher der chemischen Wahlverwandtschaften, die Goethe im Hinblick auf sein Beziehungsgeflecht wählt, die fachdidaktischen Wissenschaftsbeziehungen besser ins Bild setzt:

Diejenigen Naturen, die sich beim Zusammentreffen einander schnell ergreifen und wechselseitig bestimmen, nennen wir verwandt. An den Alkalien und Säuren, die, obgleich einander entgegengesetzt und vielleicht eben deswegen, weil sie einander entgegengesetzt sind, sich am entschiedensten suchen und fassen, sich modifizieren und zusammen einen neuen Körper bilden, ist diese Verwandtschaft auffallend genug. (Goethe 1998 [1809], S. 40)

Zwar zeigt die Metapher der gegenseitigen Modifikation von »Alkalien und Säuren« durchaus Parallelen zur beschriebenen Transdisziplinarität, so ungehindert und vollständig wie in der beschriebenen chemischen Reaktion ist aber keine der wissenschaftlichen Synthesen, wie die Projektbeschreibungen gezeigt haben. Problematisch ist auch, dass für Eduard »Verwandtschaften erst interessant [werden], wenn sie Scheidungen bewirken« (Goethe 1998 [1809], S. 40). Das ist im Hinblick auf die Fachdidaktik Deutsch und die germanistischen Fachwissenschaften schlichtweg nicht möglich und auch nicht nötig, wie Frederking und Henschel zu Recht betonen: »Literaturdidaktik und Deutschdidaktik können sich [...] - wie viele andere Fachdidaktiken zuvor - zu empirisch forschenden Disziplinen entwickeln, die dem Prinzip der Evidenzorientierung verpflichtet sind, ohne dabei ihre hermeneutischen Wurzeln zu suspendieren « (Frederking/Henschel 2016, S. 92).

Treffender scheint da eine andere Beschreibung chemischer Verbindungen aus Goethes Wahlverwandtschaften die Rolle der Fachdidaktiken im Wissenschaftsdiskurs zu charakterisieren: »[...] so gibt es auch in unserer chemischen Welt Mittelglieder, dasjenige zu verbinden, was sich einander abweist.« (Goethe 1998 [1809], S. 39). Auch wenn nicht per se unterstellt werden soll, dass sich empirische Bildungsforschung und germanistische Fachwissenschaft »abweis[en]«, so hat die Fachdidaktik Deutsch als transdisziplinäre Wissenschaft doch das Zeug dazu, solch ein verbindendes $»$ Mittelglied $« \mathrm{zu}$ sein.

Funding Open Access funding enabled and organized by Projekt DEAL.

Open Access Dieser Artikel wird unter der Creative Commons Namensnennung 4.0 International Lizenz veröffentlicht, welche die Nutzung, Vervielfältigung, Bearbeitung, Verbreitung und Wiedergabe in jeglichem Medium und Format erlaubt, sofern Sie den/die ursprünglichen Autor(en) und die Quelle ordnungsgemäß nennen, einen Link zur Creative Commons Lizenz beifügen und angeben, ob Änderungen vorgenommen wurden.

Die in diesem Artikel enthaltenen Bilder und sonstiges Drittmaterial unterliegen ebenfalls der genannten Creative Commons Lizenz, sofern sich aus der Abbildungslegende nichts anderes ergibt. Sofern das betreffende Material nicht unter der genannten Creative Commons Lizenz steht und die betreffende Handlung nicht nach gesetzlichen Vorschriften erlaubt ist, ist für die oben aufgeführten Weiterverwendungen des Materials die Einwilligung des jeweiligen Rechteinhabers einzuholen. 
Weitere Details zur Lizenz entnehmen Sie bitte der Lizenzinformation auf http://creativecommons.org/ licenses/by/4.0/deed.de.

\section{Literatur}

Abraham, Ulf (2019): Die Germanistik und das Schulfach Deutsch, oder: Keine einfache Beschreibung eines komplexen Verhältnisses! In: Didaktik Deutsch 46, S. 6-12.

Artelt, Cordula/Schlagmüller, Matthias (2004): Der Umgang mit literarischen Texten als Teilkompetenz im Lesen? Dimensionsanalysen und Ländervergleiche. In: Ulrich Schiefele/Cordula Artelt/Wolfgang Schneider/Petra Stanat (Hg.): Struktur, Entwicklung und Förderung von Lesekompetenz. Vertiefende Analysen im Rahmen von PISA 2000. Wiesbaden, S. 169-196.

Baumert, Jürgen/Kunter, Mareike (2011): Das Kompetenzmodell von COACTIV. In: Mareike Kunter/ Jürgen Baumert/Werner Blum/Ute Klusmann/Michael Neubrand (Hg.): Professionelle Kompetenz von Lehrkräften. Ergebnisse des Forschungsprogramms COACTIV. Münster, S. 29-53.

Blömeke, Sigrid, Gustaffson, Jan-Eric, Shavelson, Richard J. (2015): Beyond Dichotomies. Competence Viewed as a Continuum. In: Zeitschrift für Psychologie 223 (1), S. 1-13.

BMBF 2019: https://www.bmbf.de/de/qualitaetsoffensive-lehrerbildung-525.html (20.08.2020).

Boelmann, Jan/Klossek, Julia/König, Lisa (2020): Dossier zum Bochumer Modell literarischen Verstehens. Online verfügbar unter https://www.ph-freiburg.de/fileadmin/user_upload/2020_BoelmannKlossekK \%C3\%B6nig_Dossier_zum_Bochumer_Modell_literarischen_Verstehens.pdf, zuletzt geprüft am 08.10.2020.

Bos, Wilfried/Valtin, Renate/Hußmann, Anke/Wendt, Heike/Goy, Martin (2017): IGLU 2016: Wichtige Ergebnisse im Überblick. In: Anke Hußmann/Heike Wendt/Wilfried Bos/Albert Bremerich-Vos/ Daniel Kasper/Eva-Maria Lankes et al. (Hg.): IGLU 2016. Lesekompetenzen von Grundschulkindern in Deutschland im internationalen Vergleich. Münster u.a., S. 13-28.

Boyken, Thomas (2016): Über wissenschaftliche Verwandtschaftsverhältnisse. Versuch einer Einordung der aktuellen Entwicklungen innerhalb der Deutschdidaktik aus literaturwissenschaftlicher Sicht. In: Iris Winkler/Frederike Schmidt (Hg.): Interdisziplinäre Forschung in der Deutschdidaktik. »Fremde Schwestern « im Dialog. Berlin u.a., S. 23-41.

De La Combe, Pierre Judet (2007): Warum sich mit Sprache beschäftigen? Vorüberlegungen zu einer europäischen Bildung. Mit einem Vorwort von Gregor Vogt-Spira. Hildesheim u.a.

DIPF (Leibniz-Institut für Bildungsforschung und Bildungsinformation) (2019): Kompetenzmodelle zur Erfassung individueller Lernergebnisse und zur Bilanzierung von Bildungsprozessen. https://www. dipf.de/de/forschung/projektarchiv/spp-km (17.07.2020).

Eco, Umberto (1973): Das offene Kunstwerk. Frankfurt a. M.

Eco, Umberto (1985): Semiotik und Philosophie der Sprache. München.

Eco, Umberto (1987a): Lector in fabula. Die Mitarbeit der Interpretation in erzählenden Texten. München.

Eco, Umberto (1987b): Semiotik. Entwurf einer Theorie der Zeichen. München.

Eco, Umberto (1992): Die Grenzen der Interpretation. München u.a.

Eco, Umberto (1994): Im Wald der Fiktionen. München u.a.

Feindt, Andreas/Meyer, Hilbert (2010): Kompetenzorientierter Unterricht. Eine didaktische Schatzsuche. In: Die Grundschulzeitschrift 24/237, S. 29-33.

Fleischer, Jens/Koeppen, Karoline/Kenk, Martina/Klieme, Eckhard/Leutner, Detlev (2013): Kompetenzmodellierung: Struktur, Konzepte und Forschungszugänge des DFG-Schwerpunktprogramms. In: Zeitschrift für Erziehungswissenschaften 16, S. 5-22.

Frederking, Volker/Brüggemann, Jörn/Albrecht, Christian (2020): Ästhetische Kommunikation im Literaturunterricht. Quantitative und qualitative Zugänge zu einer besonderen Form personaler und funktionaler literarischer Bildung. In: Felix Heizmann/Johannes Mayer/Marcus Steinbrenner (Hg.): Das Literarische Unterrichtsgespräch. Didaktische Reflexionen und empirische Rekonstruktionen. Baltmannsweiler, S. 295-314.

Frederking, Volker/Henschel, Sofie (2016): Interdisziplinäre Forschung in der Deutschdidaktik am Beispiel des Projekts Literarästhetische Urteils- und Verstehenskompetenz (LUK). In: Iris Winkler/Frederike Schmidt (Hg.): Interdisziplinäre Forschung in der Deutschdidaktik. »Fremde Schwestern « im Dialog. Berlin u.a., S. 71-99.

Gaier, Lisa (i. Vorb.): Gut erklärt? FALKE-D: Eine empirische Studie zu Erklärungen im Deutschunterricht aus unterschiedlichen Beobachterperspektiven. 
Gaier, Lisa/Schilcher, Anita (i. Vorb.): Gutes Erklären im Deutschunterricht - eine empirische Studie zu unterschiedlichen Beobachterperspektiven. In: Anita Schilcher/Stefan Krauss/Alfred Lindl/Sven Hilbert (Hg.): Fachspezifische Lehrerkompetenzen im Erklären. Weinheim.

Goethe, Wolfgang von ([1809] 1998): Die Wahlverwandtschaften. Frankfurt a.M./Leipzig.

Groeben, Norbert (2006): Zur konzeptuellen Struktur des Konstrukts »Lesekompetenz«. In: Norbert Groeben/Bettina Hurrelmann (Hg.): Lesekompetenz. Bedingungen, Dimensionen, Funktionen. Weinheim/ München, S. 11-21.

Groeben, Norbert/Hurrelmann, Bettina (2006): Einleitung: Die Grundkonzeption des Weiterbildungsprogramms. In: Norbert Groeben (Hg.): Empirische Unterrichtsforschung in der Literatur- und Lesedidaktik. Weinheim/München, S. 11-30.

Grzesik, Jürgen (1990): Textverstehen lernen und lehren. Stuttgart.

Heckhausen, Heinz (1972): Discipline and Interdisciplinarity. In: Interdisciplinarity: Problems of Teaching and Research in Universities. Paris: OECD, S. 83-89.

Henschel, Sofie/Roick, Thorsten (2013): Zusammenhang zwischen Empathie und dem Verstehen literarischer Texte. In: Zeitschrift für Entwicklungspsychologie und Pädagogische Psychologie 45, S. 103-113.

Henschel, Sofie/Meier, Christel/Roick, Thorsten (2016): Effects of two types of task instructions on literary text comprehension and motivational and affective factors. In: Learning and Instruction 44, S. 11-21.

Henschel, Sofie (2013): Effekte motivationaler und affektiver Merkmale auf das Verstehen von literarischen und faktualen Texten. Diss. Berlin. https://refubium.fu-berlin.de/bitstream/handle/fub188/ 12128/Dissertation-Henschel.pdf?sequence=1\&isAllowed=y (20.08.2020).

Kintsch, Walter (1988): The Role of Knowledge in Discourse Comprehension: A Construction-Integration Model. In: Psychological Review 95 (2), S. 163-182.

Kintsch, Walter (1994): Kognitionspsychologische Modelle des Textverstehens. Literarische Texte. In: Walter Kintsch/Kurt Reusser/Marianne Reusser-Weyeneth (Hg.): Verstehen. Psychologischer Prozess und didaktische Aufgabe. Bern, S. 39-53.

Klieme, Eckhard/Leutner, Detlev (2006): Kompetenzmodelle zur Erfassung individueller Lernergebnisse und zur Bilanzierung von Bildungsprozessen. Beschreibung eines neu eingerichteten Schwerpunktprogramms der DFG. In: Zeitschrift für Pädagogik 52/6, S. 876-903.

Klieme, Eckhard/Rakoczy, Katrin (2008): Empirische Unterrichtsforschung und Fachdidaktik. Outcome-orientierte Messung und Prozessqualität des Unterrichts. In: Zeitschrift für Pädagogik 54/2, S. 222-237.

KMK = Sekretariat der Ständigen Konferenz der Kultusminister der Länder in der Bundesrepublik Deutschland (Hg.) (2003): Bildungsstandards für das Fach Deutsch für den Mittleren Schulabschluss. Beschluss vom 04.12.2003. München.

Köppe, Tilmann/Winko, Simone (2008): Neuere Literaturtheorien. Eine Einführung. Stuttgart.

Krauss, Stefan/Lindl, Alfred/Schilcher, Anita/Tepner, Oliver (2017): Das Forschungsprojekt FALKO - ein einleitender Überblick. In: Stefan Krauss/Alfred Lindl/Anita Schilcher/Michael Fricke/Anja Göhring/ Bernhard Hofmann et al. (Hg.): FALKO: Fachspezifische Lehrerkompetenzen. Konzeption von Professionswissenstests in den Fächern Deutsch, Englisch, Latein, Physik, Musik, Evangelische Religion und Pädagogik; mit neuen Daten aus der COACTIV-Studie. Münster, S. 9-65.

KVFF = Konferenz der Vorsitzenden Fachdidaktischer Fachgesellschaften (Hg.) (1998): Fachdidaktik an wissenschaftlichen Hochschulen. In: Fachdidaktik in Forschung und Lehre, S. 13-17.

Leiteritz, Christiane (2004): Hermeneutische Theorien. In: Martin Sexl (Hg.): Einführung in die Literaturtheorie. Wien, S. 129-159.

Lindl, Alfred/Krauss, Stefan/Schilcher, Anita/Hilbert, Sven (2020): Statistical Methods in Transdisciplinary Educational Research. In: Frontiers in Education 5, S. 97. https://doi.org/10.3389/feduc.2020. 00097

Magliano, Joseph P./Baggett, William B./Graesser, Arthur C. (1996): A taxonomy of inference categories that may be generated during the comprehension of literary texts. In: Roger J. Kreuz/Mary Sue MacNealy (Hg.): Empirical approaches to literature and aesthetics. Norwood, New Jersey, S. 201-220.

Meier, Christel (i. Vorb.): Kompetenzfacetten literarästhetischer Sprachreflexion. Theoretische Verortung - Empirische Analysen - Ansatzpunkte didaktischer Förderung. (Angenommene Habilitationsschrift FAU Erlangen-Nürnberg 2019).

Meier, Christel (2020a): Literarästhetische Sprachreflexion. Zur Modellierung und empirischen Überprüfung einer vernachlässigten Kompetenz an der Grenze von Sprach- und Literaturdidaktik. In: Ann Peyer/Benjamin Uhl (Hg.): Sprachreflexion - Handlungsfelder und Erwerbskontexte. Berlin u.a., S. 213-241. 
Meier, Christel (2020b): »Und wieso reimt sich bei dir alles?« - Profitieren Jugendliche von ihrer Freizeitlektüre für einen sprachreflexiven Umgang mit Literatur? In: Jörn Brüggemann/Birgit Mesch (Hg.): Sprache als Herausforderung - Literatur als Ziel. Kinder- und jugendliterarische Texte und Medien als Ressource für sprachsensibles Lernen. Teil 1. 2 Bde. Baltmannsweiler, S. 133-153.

Meier, Christel/Roick, Thorsten/Henschel, Sofie (2013): Erfassung literarischen Textverstehens. Zu Faktoren der Aufgabenschwierigkeit bei der Konstruktion von Testaufgaben. In: Carola Rieckmann/Jessica Gahn (Hg.): Poesie verstehen - Literatur unterrichten. Baltmannsweiler, S. 103-123.

Meier, Christel/Roick, Thorsten/Henschel, Sofie/Brüggemann, Jörn/Frederking, Volker/Rieder, Adelheid/ Gerner, Volker/Stanat, Petra (2017): An Extended Model of Literary Literacy. In: Detlev Leutner/Jens Fleischer/Juliane Grünkorn/Eckhard Klieme (Hg.): Competence Assessment in Education. Cham, S. 55-74.

Mittelstraß, Jürgen (2005): Methodische Transdisziplinarität. In: Technikfolgenabschätzung. Theorie und Praxis 14 (2), S. 18-23.

Pieper, Irene/Rosebrock, Cornelia/Wirthwein, Heike/Volz, Steffen (2004): Lesesozialisation in schriftfernen Lebenswelten. Lektüre und Mediengebrauch von HauptschülerInnen. Weinheim/München.

Popper, Karl (1963). Conjectures and Refutations: The Growth of Scientific Knowledge. New York.

Praetorius, Anna-Katharina/Klieme, Eckhard/Herbert, Benjamin/Pinger, Petra (2018): Generic dimensions of teaching quality: the German framework of Three Basic Dimensions. ZDM Mathematics Education, 50 (3), S. 407-426.

Roick, Thorsten/Frederking, Volker/Henschel, Sofie/Meier, Christel (2013): Literarische Textverstehenskompetenz bei Schülerinnen und Schülern unterschiedlicher Schulformen. In: Cornelia Rosebrock/ Andrea Bertschi-Kaufmann (Hg.): Literalität erfassen: bildungspolitisch, kulturell, individuell. Weinheim u. a., S. 69-84.

Rosebrock, Cornelia/Nix, Daniel (2008): Grundlagen der Lesedidaktik und der systematischen schulischen Leseförderung. Baltmannsweiler.

Rothstein, Björn (2019): Allein gelassen? Lehramtsstudierende zwischen fachwissenschaftlicher germanistischer Ausdifferenzierung und integrativem Deutschunterricht. In: Didaktik Deutsch 46, S. 13-18.

Schach, Rosmarie von ( $\left.{ }^{3} 1994\right)$ : Tochterliebe. Würzburg.

Schalk, Helge (2000): Umberto Eco und das Problem der Interpretation. Würzburg.

Schilcher, Anita/Krauss, Stefan (2019): Anforderungen an die Lehrerbildung aus fachdidaktischer Perspektive. In: Nele McElvany/Wilfried Bos/Heinz Holtappels/Annika Ohle-Peters (Hg.) Bedingen und Effekte von Lehrerbildung Lehrkraftkompetenzen und Lehrkrafthandeln. Münster, S. 29-49.

Schilcher, Anita/Krauss, Stefan/Kirchhoff, Petra/Lindl, Alfred/ Hilbert, Sven et al. (2021): FALKE: Experiences From Transdisciplinary Educational Research by Fourteen Disciplines. Frontiers in education. https://doi.org/10.3389/feduc.2020.579982.

Schneuwly, Bernhard (2009): Die Fachdidaktiken - im Zentrum der Unterrichtsforschung und -entwicklung. In: Beiträge zur Lehrerinnen- und Lehrerbildung 27 (3), S. 313-326.

Schnotz, Wolfgang/Dutke, Stephan (2004): Kognitionspsychologische Grundlagen der Lesekompetenz: Mehrebenenverarbeitung anhand multipler Informationsquellen. In: Ulrich Schiefele/Cordula Artelt/Wolfgang Schneider/Petra Stanat (Hg.): Struktur, Entwicklung und Förderung von Lesekompetenz. Vertiefende Analysen im Rahmen von PISA 2000. Wiesbaden, S. 61-99.

Schröder, Hartwig (32015): Didaktisches Wörterbuch. Wörterbuch der Fachbegriffe von »Abbilddidaktik« bis »Zugpferd-Effekt«. München/Wien.

Seidel, Tina/Shavelson, Richard (2007): Teaching Effectiveness Research in the Past Decade. The Role of Theory and Research Design in Disentangling Meta-Analysis Results. In: Review of Educational Research, 77 (4), S. 454-499.

KMK = Sekretariat der Ständigen Konferenz der Kultusminister der Länder in der Bundesrepublik Deutschland (Hg.) (2004): Bildungsstandards für das Fach Deutsch für den Primarbereich. Beschluss vom 15.10.2004. München.

KMK = Sekretariat der Ständigen Konferenz der Kultusminister der Länder in der Bundesrepublik Deutschland (Hg.) (2012): Bildungsstandards für das Fach Deutsch für die allgemeine Hochschulreife. Beschluss vom 18.10.2012. Köln.

Spinner, Kaspar H. (2012): Wie Fachwissen das literarische Verstehen stört und fördert. In: Irene Pieper/ Dorothee Wieser (Hg.): Fachliches Wissen und Literarisches Verstehen. Studien zu einer brisanten Relation. Frankfurt a.M. u.a., S. 53-69.

Steinhauer, Lydia (2010): Involviertes Lesen. Eine empirische Studie zum Begriff und seiner Wechselwirkung mit literarästhetischer Urteilskompetenz. Freiburg i.Br.

Stiller, Tanja (2017): Literarästhetische Verstehenskompetenz in der Grundschule. Baltmannsweiler. 
Terhart, Ewald (2002): Fremde Schwestern. Zum Verhältnis von Allgemeiner Didaktik und empirischer Lehr-Lern-Forschung. In: Zeitschrift für Pädagogische Psychologie 16 (2), S. 77-86.

Terhart, Ewald (2006): Kompetenzen von Grundschullehrerinnen und -lehrern. In: Petra Hanke (Hg.): Grundschule in Entwicklung. Herausforderungen und Perspektiven für die Grundschule heute. Münster, S. 233-248.

Van Dijk, Teun Adrianus/Kintsch, Walter (1983): Strategies of discourse comprehension. Orlando.

Van Holt, Nadine/Groeben, Norbert (2005): Das Konzept des Foregrounding in der modernen Textverarbeitungspsychologie. In: Journal für Psychologie 13 (4), S. 311-332.

Vipond, Douglas/Hunt, Russel A. (1984): Point-Driven Understanding: Pragmatic and Cognitive Dimensions of Literary Reading. In: Poetics 13, S. 261-277.

Winkler, Iris (2016): Was die Diskussion über Wissen im Literaturunterricht über die Literaturdidaktik verrät - oder: Für einen selbstbewussteren Umgang mit Normsetzungen. In: Thomas Möbius/Michael Steinmetz (Hg.): Wissen und literarisches Lernen. Grundlegende theoretische und didaktische Aspekte. Frankfurt a.M., S. 61-70.

Winkler, Iris/Schmidt, Frederike (2016): Interdisziplinäre Forschung in der Deutschdidaktik. Eine Zwischenbilanz. In: Iris Winkler/Frederike Schmidt (Hg.): Interdisziplinäre Forschung in der Deutschdidaktik. »Fremde Schwestern« im Dialog. Berlin u.a., S. 7-22.

Zwaan, Rolf A. (1993): Aspects of literary comprehension. Amsterdam u.a. 